\title{
Research of a Dynamic Node Data Compressive Sensing in WSN
}

\author{
Zhi Yang \\ (Zhejiang University of Water Resources and Electric Power, HangZhou \\ 310018, China) \\ zjhzyangzhi@163.com
}

\begin{abstract}
Aiming at the problem of unsolved data spatio-temporal correlation emerged during the high-speed movement of nodes in WSN network, a compressive sensing reconstruction algorithm of dynamic node data is proposed in this paper. First, estimate the sparseness of network data sparse coefficient vectors through fragmental increasing supporting set, and then the optimal supporting set element is selected in each section by supporting set backtracking optimization method. Simulation results show that this algorithm can reconstruct WSN sensor network data in the condition that sparseness of sparse coefficient vector is uncertain with the complexity of reconstruction error and measuring operation needed in reconstruction obviously lower than traditional algorithms.
\end{abstract}

Keywords: WSN; compressive sensing; reconstruction; dynamic data

\section{Introduction}

Data transmission of dynamic node in wireless sensor network has become the research hotpot of sensor network [1-2]. It's mainly because more and more sensor nodes have appeared in dynamic environment, such as vehicle-sensor, etc, and these dynamic sensor nodes transmit data mainly by wireless communication method, thus it can collect data to transmit. Due to the large scale of sensor nodes, how to correctly transmit perceived data of each node to processing center becomes the important challenge of sensor network technology of dynamic node [3]. As researchers have successively put forward many data-collection methods of dynamic sensor network, in order to reduce energy consumption of data transmission, literature [4] put forward a sparse signal reconstruction method based on compressed sensing and adaptive orthogonal matching pursuit algorithm. Literature [5] put forward sensor network data fusion method based on multi-resolution and compressed sensing. Literature [6] design a strategy based on DS evidence theory and compressed sensing WSN hybrid data fusion. Simulation experiments show this method has advantages of smaller reconstruction error and larger network lifetime. Literature [7] put forward a data-collection method based on delay-bound rooting protocols. Most of these methods require data output end has strong data processing and storage capacity, as it not only require higher demands of dynamic node computing power. But it doesn't take into the account of the problem of data dependency of each node.

Compressed sensing theory [8-11] provides ideal solution for the data-collection problem of wireless sensor network. Compressed sensing theory can make full use of computing power asymmetry of data sending and receiving ends, which can maximize system sensing and computing power and make effective use of node data dependency to increase data reconstruction accuracy [12-14]. This paper put forward compressed sensing reconstruction algorithm based on dynamic node data. The algorithm evaluates sparse degree of sparse coefficient vector of network data step by step using support set potential segment increasing method. While in each segment, determine nonzero element location and amplitude in sparse vector by using support set retrospective optimization method. 
Compared with past algorithms, algorithm in this paper can reconstruct accurately when network data dependency degree is uncertain, and reconstruction error and measurement computing complexity required by construction degrease significantly.

\section{Problem Description}

Suppose the number of several dynamic sensors of each node is $J$, during data-collection process of each node, repeat $n$ times of sampling processes, and every node data length is $n$. Mark collected signal node data as matrix column, thus network data can be presented as

$$
\boldsymbol{X}_{2 D}=\left(\begin{array}{ccc}
x_{11} & \cdots & x_{1 J} \\
\vdots & \ddots & \vdots \\
x_{n 1} & \cdots & x_{n J}
\end{array}\right)
$$

For the ease of presentation, connect data of each node, and network data can be written as vector form

$$
\boldsymbol{X}=\operatorname{vec}\left(\boldsymbol{X}_{2 D}\right)=\left(x_{11}, \ldots, x_{1 n}, x_{12}, \ldots, x_{n 2}, x_{1 J}, \ldots, x_{n J}\right)^{T}=(x(1), x(2), \ldots x(N))^{T}
$$

$$
\text { 其中 } \boldsymbol{X} \in R^{N}, N=J \times n \text { 。 }
$$

Among which, $\boldsymbol{X} \in R^{N}, N=J \times n$

Before compressed sensing measurement of data, sparse convert the signal by Discrete Cosine Transform

$$
\boldsymbol{X}=\boldsymbol{\Psi} \boldsymbol{s}=\sum_{i=1}^{N} \psi_{i} s_{i}=\sum_{k=1}^{K} \psi_{k} s_{k}, \quad s_{k} \neq 0
$$

Among which, $\boldsymbol{\Psi}$ is $N \times N$ dimension DCT transformation matrix, $N$ dimension sparse coefficient vector $s_{k}$ includes $K(K<<N)$ nonzero elements. Measurement matrix used to measure data produces in each node, mark the measurement matrix produced in node $j(j \in 1,2, \ldots, J)$ as $\boldsymbol{\Phi}_{j}$, matrix dimension is $m \times n(m<<n)$. Measured value of each node can be presented in the below vector form:

$$
\boldsymbol{Y}=\left(y_{11}, \ldots, y_{1 m}, y_{12}, \ldots, y_{m 2}, y_{1 J}, \ldots, y_{m J}\right)^{T}=(y(1), y(2), \ldots y(M))^{T}
$$

Thus measurement process of network data can be presented in the below matrix vector form:

$$
\boldsymbol{Y}=\boldsymbol{\Theta} \boldsymbol{X}=\boldsymbol{\Phi} \boldsymbol{\Psi}
$$

Among which, $\boldsymbol{Y} \in R^{M}, M=J \times m$, measurement matrix

$$
\boldsymbol{\Phi}=\left(\begin{array}{cccc}
\Phi_{1} & 0 & \cdots & 0 \\
0 & \Phi_{2} & \cdots & 0 \\
\vdots & \vdots & \ddots & \vdots \\
0 & 0 & \cdots & \Phi_{J}
\end{array}\right) \in R^{M \times N}
$$

In order to realize network data recovery of WSN sensor, under condition with known measurement $Y$ and recovery matrix $\boldsymbol{\Theta}$, reconstruct sparse coefficient vector. However, sparse degree of sparse coefficient vector $\boldsymbol{s}$ of dynamic node data in sensor network changes with node mobility, so traditional reconstruction method doesn't adapt anymore. 
The paper put forward a new network data reconstruction algorithm.

\section{Dynamic Node Data Reconstruction Algorithm}

\subsection{Algorithm Description}

Know from compressed sensing theory, network data reconstruction realizes data construction mainly by determining nonzero element location and amplitude step by step in sparse coefficient vector. Based on convert reconstruction algorithms, the paper determines support set step by step in segment increasing method, detailed steps is shown as below:

Initialization: residual error $\boldsymbol{r}_{\boldsymbol{0}}=\boldsymbol{Y}$, iteration times $t=1$, support set potential iteration step length size $\neq 0$, support set potential $L=$ size, segment sequence stage $=1$, index value set $\Lambda_{0}=\phi, \Omega_{0}=\phi$.

(1)Compute correlation coefficient of column and residual of recovery matrix, and choose $2 L$ numbers of correlation coefficient with maximum amplitude.

$$
u_{t}=\left\{u_{i}\left|u_{i}=\right|\left\langle r, \Theta_{i}\right\rangle \mid\right\} i=1,2, \ldots N, \Omega_{t_{0}}=\left(u_{t}, 2 L\right)
$$

(2)Update index value set, and choose $L$ numbers of correlation coefficient with maximum amplitude

$$
\Lambda_{t_{0}}=\Lambda_{t-1} \cup \Omega_{t}, \quad \Lambda_{t}=\left(\Lambda_{t_{0}}, L\right)
$$

(3)Estimate sparse coefficient vector

$$
\hat{\boldsymbol{s}}=\arg \min _{i \in R^{\Lambda_{t}}}\left\|\boldsymbol{Y}-\boldsymbol{\Theta}_{\boldsymbol{A}_{t}} \boldsymbol{s}\right\|_{2}
$$

(4)Update residual error: $\boldsymbol{r}_{\boldsymbol{t}}=\boldsymbol{y}-\boldsymbol{\Theta}_{\boldsymbol{A}_{t}} \hat{\boldsymbol{s}}$

(5)Judge algorithm termination condition: $\left\|\hat{\boldsymbol{s}}_{\boldsymbol{t}-\boldsymbol{I}}-\hat{\boldsymbol{s}}_{\boldsymbol{t}}\right\|_{2}<\varepsilon$. If it satisfy, terminate algorithm, output $\hat{\boldsymbol{x}}=\boldsymbol{\Psi} \hat{\boldsymbol{s}}$; if it doesn't satisfy, enter into step (6).

(6)Judge segment termination condition: $\left\|\boldsymbol{r}_{\boldsymbol{t}}\right\|_{2} \geq\left\|\boldsymbol{r}_{\boldsymbol{t}-\boldsymbol{1}}\right\|_{2}$. If it satisfy, enter into step (7); if it doesn't satisfy, enter into step (8).

(7)Begin next segment iteration: support set potential $L=L+$ size, $t=t+1$, stage $=$ stage $+1, \quad$ enter into step $(1)$.

(8)Continue this segment iteration: $t=t+1$, enter into step (1).

Final solution: $\hat{\boldsymbol{x}}=\boldsymbol{\Psi} \hat{\boldsymbol{s}}$

\subsection{Algorithm Convergent Analysis}

To test effectiveness and reliability of this algorithm, this section derivate and prove the algorithm convergence theoretically. $\Lambda^{\prime}$ represents index value selected after $n$ times of iteration, $\Lambda_{n}$ represents index value set after $n$ times of iteration, $F_{1}$ represents index value set used to signal reconstruction after $n$ times of iteration, $F_{2}$ represents index value set that failed to be selected after $n$ times of iteration, $F$ represents index value set used for final signal reconstruction. Combining algorithm in last section, we can know that, the above sets have below relations:

$T_{1} 、 T_{2}$ separately represent potential of set $F_{1} 、 F_{2}$, as $T_{1}=\left|F_{1}\right|, T_{2}=\left|F_{2}\right|$. After $n$ times of iteration, residual error can be written as: 


$$
r_{n}=Y-Q_{A_{n}}(Y)=\Theta_{F_{2}} s_{F_{2}}-Q_{\Lambda_{n}}\left(\Theta_{F_{2}} s_{F_{2}}\right)=\Theta_{F_{2}} s_{F_{2}}-Q_{\Lambda_{n}} s_{Q}
$$

Among which, $\boldsymbol{Q}_{\Lambda_{n}}(\boldsymbol{Y})$ represent the projection of corresponding element on $\Lambda_{n}$. Note $\boldsymbol{\Theta}_{A_{n}}^{T} \boldsymbol{\Theta}_{A_{n}}$ is reversible, can get $\boldsymbol{s}_{Q}=\left(\boldsymbol{\Theta}_{A_{n}}^{T} \boldsymbol{\Theta}_{A_{n}}\right)^{-1} \boldsymbol{\Theta}_{A_{n}}^{T} \boldsymbol{\Theta}_{F_{2}} \boldsymbol{S}_{F_{2}}$

Thus we have

$$
\left\|\Theta_{\lambda^{\prime}}^{T} r_{n}\right\|=\left\|\Theta_{A^{\prime}}^{T}\left(\Theta_{F_{2}} S_{F_{2}}-Q_{\Lambda_{n}} S_{Q}\right)\right\| \leq\left\|\Theta_{A^{\prime}}^{T} \Theta_{F_{2}} S_{F_{2}}\right\|+\left\|\Theta_{\lambda^{\prime}}^{T} Q_{\Lambda_{n}} S_{Q}\right\|
$$

Known from algorithm, number of index value failed to be selected isn't bigger than step support set potential iteration step length size, therefore, known from (5) formula and (6) formula

$$
\left\|\Theta_{A^{\prime}}^{T} \boldsymbol{r}_{n}\right\| \geq\left\|\Theta_{F_{2}}^{T} \boldsymbol{r}_{n}\right\| \geq\left\|\Theta_{F_{2}}^{T} \Theta_{F_{2}} \boldsymbol{s}_{F_{2}}\right\|-\left\|\Theta_{F_{2}}^{T} \Theta_{A_{n}} \boldsymbol{s}_{Q}\right\|
$$

Note that recovery matrix $\boldsymbol{\Theta}$ meet Restricted Isometry Property, as for any $1<\delta<0$, sparse vector $s$ have

$$
(1-\delta)\|\boldsymbol{s}\|^{2} \leq\|\boldsymbol{\Theta} \boldsymbol{s}\|^{2} \leq(1+\delta)\|\boldsymbol{s}\|^{2}
$$

Satisfy, thus $\boldsymbol{\Theta}_{A_{n}}$ also meet RIP, in combination with formula (7) can get

$$
\left\|\Theta_{\Lambda^{\prime}}^{T} \boldsymbol{r}_{n}\right\| \geq(1-\delta)\left\|\boldsymbol{S}_{\boldsymbol{F}_{2}}\right\|-\left(\frac{\delta^{2}}{1-\delta}\right)\left\|\boldsymbol{S}_{F_{2}}\right\|
$$

Suppose $\delta<1 / 3$, thus have

Thus we know

$$
\delta\left\|\boldsymbol{S}_{F_{2}}\right\|+\left(\frac{\delta^{2}}{1-\delta}\right)\left\|\boldsymbol{S}_{F_{2}}\right\|>(1-\delta)\left\|\boldsymbol{S}_{F_{2}}\right\|-\left(\frac{\delta^{2}}{1-\delta}\right)\left\|\boldsymbol{S}_{F_{2}}\right\|
$$

Algorithm convergence can be illustrated by this.

\section{Experiment Simulation}

In this paper, in Windows xp system, CPU is core 13 , memory is 4GDDR3, and hard disk is $500 \mathrm{G}$, simulation software adopts Matlab2012. The paper first reconstruct network data by using general sparse vector to test accuracy of algorithm in this paper; second, reconstruct collected network data to test effectiveness of the algorithm.

Compressed sensing reconstruction algorithm requires the measured data meet certain property. In order to let simulation results can reflect algorithm property, measure reconstruction sparse data by BRMM matrix to produce sparse vector. This paper choose sparse vector length $N 256$, number of measured values meet $M / N=0.4$. Under same simulation condition, reconstruct same sparse vector by traditional algorithms as SP algorithm, Cosamp algorithm and Stomp algorithm. Adopt relative error formula

$$
\eta=\frac{\|\hat{x}-x\|_{2}}{\|x\|_{2}}=\frac{\sqrt{\sum_{i=0}^{N-1}\left(\hat{x}_{i}-x_{i}\right)^{2}}}{\sqrt{\sum_{i=0}^{N-1}\left(x_{i}\right)^{2}}}
$$


Formula (10) is judge standard of reconstruction, repeat 200 times of experiments under every sparse condition, regard average value of reconstruction error as final error under this spars condition. Simulation results show as Figure 1.

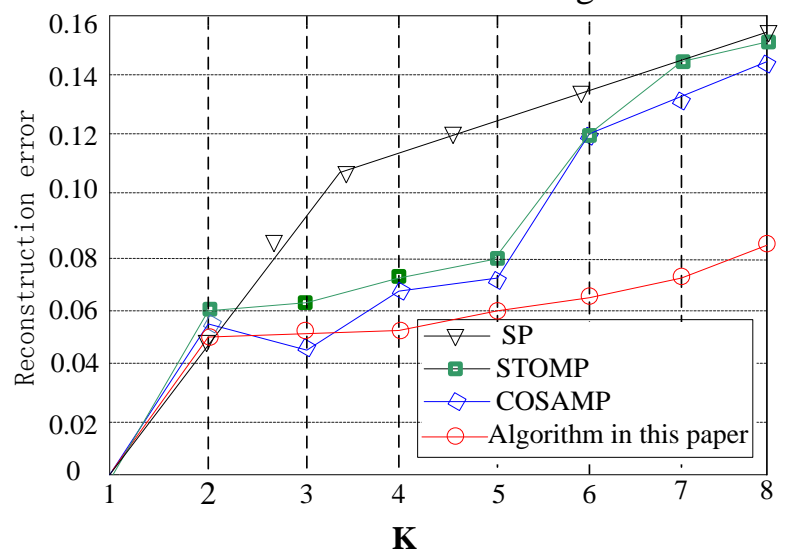

Figure 1. Reconstruction Error Comparison

Known from the results, when reconstruction vector sparse degree $K$ is lower, each algorithm reconstruction error is similar, but as $K$ increases, traditional algorithm reconstruction error is obviously higher than the algorithm in this paper. If define reconstruction failure probability $P_{e r}=\frac{\operatorname{num}\left(\max (\hat{x}-x)>x^{*} 0.1\right)}{n u m_{\text {total }}}$, reconstruction failure probability result shown in Figure 2 can further test that algorithm in this paper can accurately reconstruct sparse vector when sparse degree of vector changes.

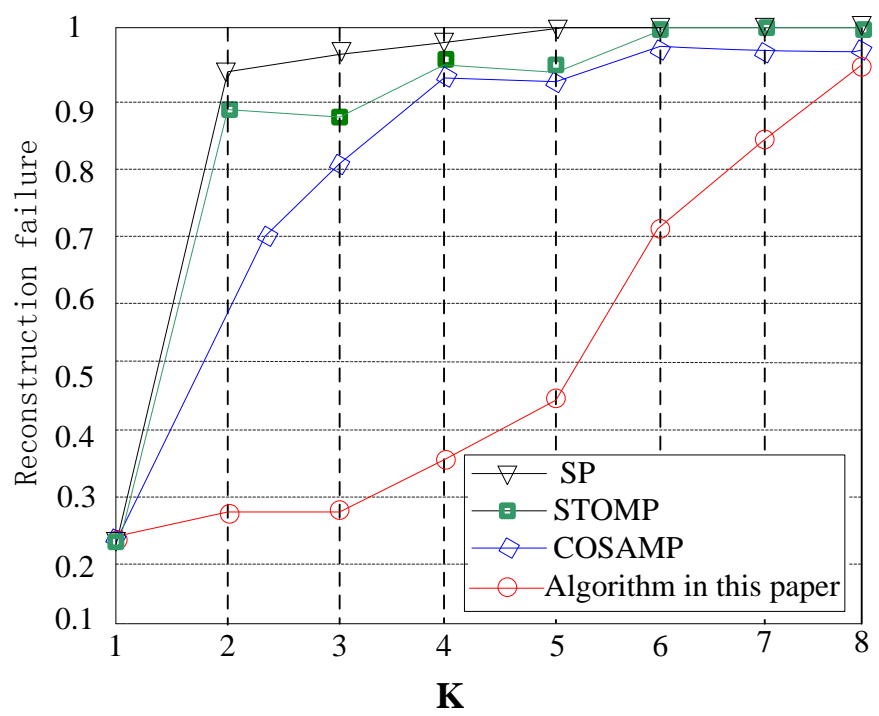

Figure 2. Reconstruction Failure Probability Comparison 


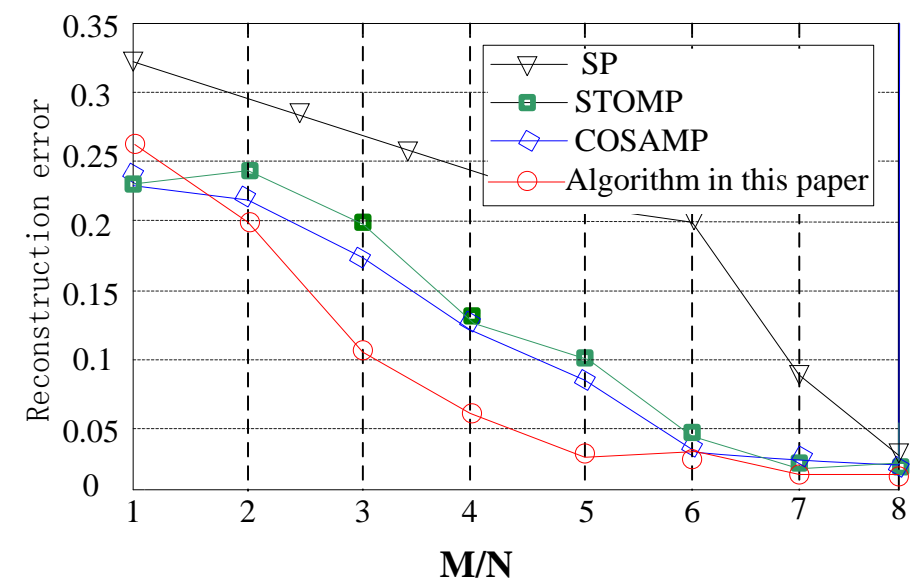

Figure 3. Reconstruction Error Comparison

The above experiments use same number of measured values; however, number of measured value also influences reconstruction error. In order to display reconstruction capacity of each algorithm under different numbers of measured values, the test chooses sparse degree $K=6$, change measured values number, make $M / N$ changes within 0.1-0.8. Also, use relative error as judgment standard of reconstruction quality. Test results are shown as Fig. 3 and Fig. 4. For the same reconstruction error, number of measured values required by algorithm in this paper is obviously smaller than that of traditional algorithm, while use same measured value number, algorithm in this paper can realize better reconstruction quality than traditional algorithm.

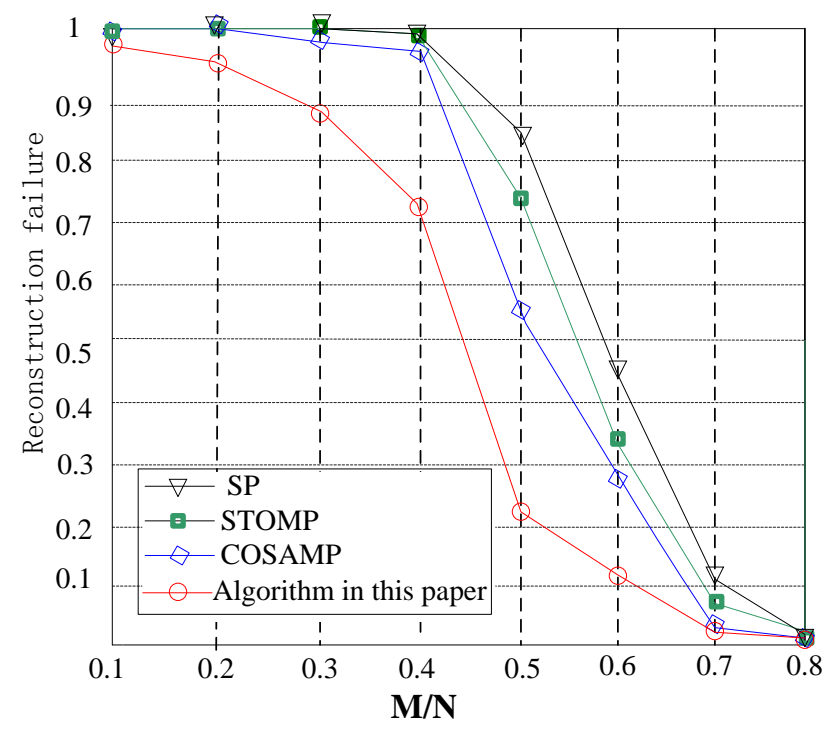

Figure 4. Reconstruction Failure Probability Comparison

\section{Conclusion}

As traditional network data compressed sensing algorithm has higher requirement for data dependency certainty degree, so it can't adapt to the practical application background of dynamic node in wireless sensor. Dynamic node data compressed sensing algorithm put forward by this paper can significantly improve dynamic node data reconstruction accuracy in sensor network, meanwhile it can reduce measuring and computing complexity required by reconstruction. Algorithm in this paper provides a reference research direction for the dynamic node development in wireless sensor. 


\section{References}

[1] Y. Charfi, N. Wakamiya and M. Murata, "Challenging issues in wireless sensor networks [R]", Advanced Network Architecture Laboratory, Osaka University, (2007).

[2] U. Lee and M. Gerla, "A survey of urban wireless sensing platforms [J]”, Computer Networks, vol. 54, no. 4, (2010), pp. 527-544.

[3] J. Mohammad, M. Rama and B. Praveen, "Wireless ad hoc and sensor networks: principles and challenges [J]", International Journal of Ad hoc, Sensor \& Ubiquitous Computing, vol. 12, no. 2, (2012), pp. 38-49.

[4] Z. Zhang, "Sparse Signal Reconstruction Based on Compressed Sensing and Adaptive Orthogonal Matching [J]", Computer Measurement \& Control, vol. 22, no. 5, (2014), pp. 1568-1571.

[5] H. Zhang, G. Liu and S. Chen, "Data Aggregation Scheme Based on Multi-Resolution and Compressive Sensing in Wireless Sensor Networks [J]", Computer Measurement \& Control, vol. 22, no. 7, (2014), pp. 2335-2338.

[6] C. Liao, "Design of Data Fusion Strategy in Wireless Sensor Network Based on DS Evidence Theory and Compressing Sensing [J]", Computer Measurement \& Control, vol. 22, no. 8, (2014), pp. 2618-2622.

[7] C. Claudio, P. Fabio and M. Ruiz, "Delay-bounded data gathering in urban vehicular sensor networks [J]", Pervasive and Mobile Computing archive, vol. 8, no. 2, (2012), pp. 180-193.

[8] Y. S. Jeong, Y. H. Han and J. J. Park, "MSNS: mobile sensor network simulator for area overage and obstacle avoidance based on GML", EURASIP Journal on Wireless Communications and Networking, vol. 12, no. 1, (2012), pp. 1-15.

[9] E. Candes, J. Romberg and T. Tao, "Robust Uncertainty Principles: Exact Signal Reconstruction From Highly Incomplete Frequency Information [J]", gg7 IEEE Transaction on Information Theory, vol. 26, no. 4, (2010), pp. 381-400.

[10] W. Shao and Z. Wei, "Advances and perspectives on compressed sensing theory [J]", Journal of Image and Graphics, vol. 17, no. 1, (2012), pp. 1-10.

[11] L. Jiaon, S. Yang and F. Liu, "Development and Prospect of Compressive Sensing [J]", Acta Electronica Sinica, vol. 20, no. 7, (2011), pp. 1651-1662.

[12] W. Chen and O. Milenkovic, "Subspace pursuit for compressive sensing: Closing the gap between performance and complexity [J]", IEEE Transaction on Information Theory, vol. 34, no. 5, (2012), pp. 2230-2249.

[13] D. Donoho, Y. Tsaig and S. Jean-Luc, "Sparse solution of underdetermined linear equations by stagewise orthogonal matching pursuit [R]", Technical Report, (2011).

[14] A. Sankaranarayanan, P. Turaga and R. Baraniuk, "Compressive Acquisition of Dynamic Scenes [C]", European Conference Company Vision, (2010), Crete, Greece.

[15] U.S. Geological Survey, Landsat Thematic Mapper [OL], Available: https://lta.cr.usgs.gov/TM.

\section{Author}

Zhi Yang ( 1976.08- ) ,male, master, instructor, research direction: computer network information. 
International Journal of Future Generation Communication and Networking Online Vol. 9, No. 4, (2016) 